\title{
A Review of Activity Time Distributions in Risk Analysis
}

\author{
Enobong Francis Udoumoh'1, Daniel W. Ebong² \\ ${ }^{1}$ Department of Mathematics/Statistics/Computer Science, University of Agriculture, Makurdi, Nigeria \\ ${ }^{2}$ Department of Mathematics and Statistics, University of Port Harcourt, Port Harcourt, Nigeria \\ Email: udoumoh.francis@uam.edu.ng,daniel.ebong@uniport.edu.ng
}

How to cite this paper: Udoumoh, E.F. and Ebong, D.W. (2017) A Review of Activity Time Distributions in Risk Analysis. American Journal of Operations Research, 7, 356-371.

https://doi.org/10.4236/ajor.2017.76027

Received: October 22, 2017

Accepted: November 24, 2017

Published: November 27, 2017

Copyright () 2017 by authors and Scientific Research Publishing Inc. This work is licensed under the Creative Commons Attribution International License (CC BY 4.0).

http://creativecommons.org/licenses/by/4.0/

\section{(c) (i) Open Access}

\begin{abstract}
Project Evaluation and Review Technique (PERT) alongside recent modifications is a popular and useful tool in project risk analysis. Over the past seven decades, there have been some modifications in PERT owing to the shift from beta distributed activity times to other activity time distributions. This paper presents a review of activity time distributions in risk analysis as found in literature up to date.
\end{abstract}

\section{Keywords}

PERT, Project Network, Activity Duration, Probability Distributions

\section{Introduction}

Project Evaluation and Review Technique (PERT) is widely used by project managers and practitioners as the probabilistic form of the Critical Path Method (CPM). The PERT method is not only useful for the estimation of project completion times but it is also workable and cost-effective for management of projects [1]. PERT has become of interest to management practitioners because of its simplicity, and flexibility to accommodate stochastic activity times. PERT was invented in 1958 for the POLARIS Missile Program by the Program Evaluation branch of the Special projects Office of US Navy [2]. Malcom's PERT network, henceforth referred to as classical PERT network, assumes that all activities are independent random variables, having approximately beta distributions parameterized by three times estimates: the optimistic time $a$, the pessimistic time $b$, and the most likely time $m$. The expected time of each activity is obtained using the formula $(a+4 m+b) / 6$ and standard deviation presented as one sixth of the range of the distribution resulting to $(b-a)^{2} / 36$ as the va- 
riance. The critical path is then computed as the longest path. With the belief that the network is large enough, the central limit theorem is applied to estimate the project completion time. For detail application of PERT, interested readers are referred to [1] [2].

Although it is well accepted that the classical PERT gives useful estimates, its assumption introduces some potential sources of bias which results in the underestimation of project completion time [3] [4] [5]. Sources of PERT bias as documented in literature include: the misspecification of the activity time distribution, the method of computing critical path by ignoring the near critical activities, and the possible violation of the normality assumption during the estimation of project completion time. There exist a sharp divide among researchers with respect to the need for introduction of new activity duration distributions in PERT. For instance, earlier works by Clark [6], Kamburowski [7] supported the use of Beta distribution. Recently, Hajdu and Bokor [8] argued still in support of the Beta distribution. They considered the following "hypothetical" judgemental estimates: 60-optimistic, 100-most likely, and 150-pessimistic whose skewness coefficient is only 0.09826 . The beta distribution defined by this coefficient of skewness is the one that approximates the normal distribution which is also supported by PERT-Beta approximation. Hence, there is no doubt about their conclusion. In spite of this, the graphical results that compared the PERT-Beta, triangular and uniform distributions still showed some obvious variations. It is possible that a set of data with much longer tail would have yielded worse results. Moreover, a comparison done with many more activity time distributions would have given more in-depth revelations. On the other hand, some researchers opine that the adoption of Beta distribution was intuitive as there was no empirical evidence for its usage. For instance, it has been demonstrated by MacCrimmon and Ryavec [3] that the error for classical PERT calculated mean and standard deviation could be up to $33 \%$ and $16 \%$ respectively. This point was buttressed by Williams [9] using simulation and graphical approaches to demonstrate the extent of discrepancies that exist between the beta and triangular distributions in the estimation of activity time parameters. Hahn and Martín [10] support the use of a more robust distribution that can accommodate outlying events. An undisputed observation in project management is that most activity time distributions are right skewed [11] [12] [13].

The importance of probability distributions in PERT cannot be overemphasized as both the simulation and analytical approaches assume probability distributions for activity durations a priori or a posteriori [5] [14] [15]. Consequently, researchers have suggested various activity duration distributions for the analyses of project networks. Unfortunately, most basic texts in operations research and project management present only PERT-beta distribution without any mention of other activity time distributions.

In this paper, we present a review of the various activity duration distributions that have been used for the analysis of project networks. Some modified versions 
of PERT-Beta approach are also presented. We further highlight the various methods adopted for parameter estimation based on these distributions.

\section{Activity Time Distributions in Literature}

\section{1) The Beta Distribution}

The originators of classical PERT [2] assumed that project activity time follows the generalized beta distribution with probability density function

$$
f(x)=\frac{\Gamma(\alpha+\beta)}{\Gamma(\alpha) \Gamma(\beta)} \frac{(x-a)^{\alpha-1}(b-x)^{\beta-1}}{(b-a)^{\alpha+\beta-1}} ; a<x<b, \alpha, \beta>0
$$

where $\alpha$ and $\beta$ are the shape parameters, $\Gamma($.$) is the gamma function. The$ mean, variance and skewness are respectively given as

$$
\mu_{x}=a+(b-a) \frac{\alpha}{\alpha+\beta}, \quad \sigma_{x}^{2}=(b-a)^{2} \frac{\alpha \beta}{(\alpha+\beta)^{2}(\alpha+\beta+1)}
$$

and

$$
\gamma_{1}=\frac{2(\beta-\alpha) \sqrt{\alpha+\beta+1}}{(\alpha+\beta+2) \sqrt{\alpha \beta}}
$$

In classical PERT, the mean and variance were estimated to be $\hat{\mu}_{x}=\frac{a+4 m+b}{6}$ and $\hat{\sigma}_{x}^{2}=\frac{(b-a)^{2}}{36}$. A study by Farnum and Stanton [16] revealed that the mean of the beta distribution in classical PERT is appropriate within some range of modal values, namely, $a+0.13(b-a)<m<b-0.13(b-a)$. This means that the estimate performs poorly outside this interval. This can either happen when the most likely estimate, $m$, is chosen to be very close to the two extreme values, $a$ and $b$, (less than $13 \%$ of the range from either $a$ or $b$ ). In other words the classical PERT estimate fails when activity time distributions are heavily tailed. Moreover, previous works reveal that the classical PERT assumptions of the mean and variance restrict us to only three members of the beta family, namely, 1) $\alpha=\beta=4$; 2) $\alpha=3-\sqrt{2}, \beta=3+\sqrt{2}$; 3) $\alpha=3+\sqrt{2}, \beta=3-\sqrt{2}$. In which case the skewness will be $0, \frac{1}{2}$ and $-\frac{1}{2}$ respectively [17] [18] [19]. This restriction led to various modifications on the classical PERT to accommodate more members of the beta family. We will discuss some of these modifications. Most of these modifications are based on the adjustments of the parameters of beta distribution.

Gollenko-Ginzburg [20] worked on the improvement of the classical PERT estimates based on only two subjective estimates the pessimistic 1) and optimistic 2) times. He posited that analysis of many project networks with lengthy periods reveals that the most likely activity time is practically useless. He pointed out that its relative location in time interval $[a, b]$ is usually close to the point $m=\frac{2 a+b}{3}$. Given the density function $f(x)=\frac{\Gamma(p+q+2)}{\Gamma(p+1) \Gamma(q+1)} x^{p}(1-x)^{p\left(m_{x}-1\right)}$ 
( $m_{x}=$ mode of $\left.x\right)$ which was obtained after a re-parametisation of the standard beta distribution, with additional assumption that $p+q \cong Z$ (constant). The following results $\mu_{y}=\frac{2 a+9 m+2 b}{13}$ and $\sigma_{y}^{2}=\frac{(b-a)^{2}}{1268}\left[22+81\left(\frac{m-a}{b-a}\right)-81\left(\frac{m-a}{b-a}\right)^{2}\right]$ were obtained for the estimation of the mean and variance of activity distribution. He showed that these formulae provide better results as compared to the classical pert formulae when the estimated mode is located in the tails of the distribution. These formulae were further reduced to $\mu_{y}=0.2(3 a+2 b)$ and $\sigma_{y}^{2}=0.04(b-a)^{2}$ on the basis of the earlier assumption of the mode, $\cong \frac{2 a+b}{3}$. A similar modification was carried out by Shankar and Sireesha [21] on the classical PERT. The approximation was achieved by their so called generalization of the assumptions on the parameters of the classical PERT method. Given the density function, $f(x)=\frac{\Gamma(p+q+2)}{\Gamma(p+1) \Gamma(q+1)} x^{p}(1-x)^{p\left(m_{x}-1\right)}$ with the relation $p+q \cong K$ (constant). Also, substituting $p+1$ and $q+1$ for $p$ and $q$ respectively they obtained the results $\mu_{x}=\frac{17 m_{x}+5}{27}$ and $\sigma_{x}^{2}=\frac{\left(17 m_{x}+10\right)\left(27-17 m_{x}\right)}{2300}$ which give $\mu_{x}=\frac{5 a+17 m+5 b}{27}$ and $\sigma_{x}^{2}=\frac{(17 m-27 a+10)(27 b-10 a-17 m)}{2300}$ for the general beta distribution. Their method further created allowance for the accommodation of some events in the tail of the distribution. Trout [22] considered a modification of the classical PERT method by replacing the most likely time (Mode) with the median. Other approximations and extensions on classical PERT are widely documented in literature [8] [16] [18] [23]-[31].

\section{2) The Normal Distribution}

The proponents of the normal activity time distribution posit that activity times can as well be normally distributed regardless of the popular opinion of the right skewed activity times. A random variable $X$ is said to be normally distributed with mean $(\mu)$ and variance $\left(\sigma^{2}\right)$ if the probability density function is given as $f(x)=\frac{1}{\sqrt{\sigma^{2} 2 \pi}} \mathrm{e}^{\frac{(x-\mu)^{2}}{2 \sigma^{2}}} ;-\infty<x<\infty$. Its coefficient of skewness is zero. Kamburowski [32] assumed that the activity durations of PERT network are independent and normally distributed random variables. He obtained a lower and upper bounds for the expected project completion time using a simple recursive algorithm. The tightness of the bounds was examined for some numerical examples. It was apparent that the lower bounds are tighter than the estimates of the classical PERT procedure when empirical activity distribution is symmetric but otherwise when the distribution is asymmetric. Kamburowski's method [32] followed after Dodin [33]. Sculli [34] proposed an approximation for the completion time mean and variance of PERT networks. His method assumed that 
activity durations are normally and independently distributed. He further assumed that various paths of the network are independent, and that the network can be transformed into the type where only maximum of two activities terminate on the same event, such that the problem of finding the distribution of $T=\max \left(N_{1}, N_{2}\right)+N_{3} . N_{i}, i=1,2,3$ are independent normal random variable with mean $\mu_{i}$ and common variance $\sigma^{2}$. He demonstrated that his method which adopts path independence produced better results than the classical PERT method which assumes complete dependence of paths. Drezner and Anklesaria [35] also developed a method for solving PERT networks as a multivariate problem taking into consideration path correlation. They assumed that each path duration is the sum of activities on the path, and then defined $T_{k} ; k=1,2, \cdots, m$ to be the duration of path. They assumed that the set of all $T_{k} ; k=1,2, \cdots, m$ follow a multivariate normal distribution, and gave the probability of completing the project in time $T$ as $P\left(T_{k} \leq T\right) ; k=1,2, \cdots, m$ resulting to an $m$-dimensional integral problem. Their method was not popular because of much computation time required for an approximate solution to be obtained even for small project networks. Cottrell [36] developed a simplified version of PERT using normally distributed activity times. The simplification was obtained by reducing the number of estimates required for activity durations from three, as in classical PERT, to two (the most likely- $m$, and the pessimistic- $b$ times) which were subjectively chosen. In such case, the most likely time $(m)$ coincided with the mean, and the variance was obtained using $\sigma_{90}^{2}(T)=\left(\frac{b-m}{1.645}\right)^{2}$. Although his method seemed to reduce the effort needed to apply PERT, it was subject to errors greater than $10 \%$ when the skewness of the actual distribution is greater than 0.28 or less than -0.48 . Kotiah and Wallace [37] also considered a doubly truncated normal distribution for the activity time distribution in PERT via a maximum entropy approach.

\section{3) The Exponential Distribution}

The exponential distribution has been used to describe activity times. Magott and Skudlarski [38], Abdelkader and Mouhamed [39] used the exponential distribution as a representation of activity duration in Stochastic activity Networks (SANs). A random variable $X$ with scale parameter $\lambda$ is said to be exponential if the probability density function is given as $f(x)=\lambda \mathrm{e}^{-\lambda x} ; x>0, \lambda>0$. Its mean variance, and skewness are $\mu_{x}=\frac{1}{\lambda}, \sigma_{x}^{2}=\frac{1}{\lambda^{2}}$, and $\gamma_{1}=2$ respectively. Abdelkader [40] later presented an adjustment to the recursive method of determining the moments of the project completion times in SANs when activity times are exponentially distributed. But one of the criticisms of using the exponential distribution is that it assumes a constant probability of completion in the next time period, irrespective of the elapsed activity duration. Hence, Abd-el-Kader [41] used the truncated exponential distribution as the activity time distribution. He equally adopted Stochastic Activity Networks (SANs) approach to obtain the moments of the project completion time. His effort yielded an improvement on 
the estimates obtained using the untruncated exponential distribution. Cinicioglu \& Shenoy [42] described how a stochastic PERT network can be transformed into a mixture of truncated exponentials Bayesian network. They adopted the Lauretsen-Jensen algorithm for solving mixtures of Guassian (MoG) hybrid bayesian networks and further approximated a PERT Bayesian network by MoG Bayes net. Their method suffered a setback during arc reversal in complex activity networks. Azaron and Modarres [43] transformed a dynamic PERT network with exponential activity duration in into stochastic network and then obtained the project completion time by constructing Continuous Time Markov Chain (CTMC). Other works on exponentially distributed activity times could be found in Kamburowski [44], Kulkarni and Adlakha [45] and Kwon, et al. [46].

\section{4) The Weibull Distribution}

The Weibull distributed activity time was considered by Abd-El-Kader [47], with density $f(x)=\beta \alpha \mathrm{e}^{-\alpha x^{\beta}} x^{\beta-1} ; x \geq 0 ; \alpha, \beta>0$. The moment method was developed for the estimation of the parameters of the stochastic activity networks (SANs). A desirable property of the Weibull distribution over the exponential distribution is that of a broad variety of monotone increasing hazard rate when the shape parameter is greater than one. McCombs et al. [48] also used the Weibull distribution to describe activity times. Their method was based on three judgmental estimates: $x_{a}, x_{b}$ being the lower and upper expert percentile estimates, and $m$ the most likely estimate. They made effort to obtain what they called exact estimates of the mean and variance of the activity distribution. A Weibull distributed random variable $X$ with density $(x)=(\beta / \theta)(x / \theta)^{\beta-1} \mathrm{e}^{-(x / \theta)^{\beta}}$; $x \geq 0 ; \theta, \beta>0$, where $\theta$ is the scale parameter and $\beta$ is the shape parameter, has mean, variance and skewness given as $\mu_{x}=\theta \Gamma\left(1+\frac{1}{\beta}\right)$, $\sigma_{x}^{2}=\theta^{2}\left[\Gamma\left(1+\frac{2}{\beta}\right)-\left(\Gamma\left(1+\frac{1}{\beta}\right)\right)^{2}\right]$ and $\gamma_{1}=\Gamma\left(1+\frac{3}{\beta}\right) \theta^{3}-3 \mu_{x} \sigma_{x}^{2}-\mu_{x}^{3}$ respectively.

\section{5) The Lognormal Distribution}

A random variable $X$ is lognormal if the probability density function is given as

$$
f(x)=\frac{1}{x \sqrt{2 \pi \sigma}} \mathrm{e}^{-\frac{(\operatorname{In} x-\mu)^{2}}{2 \sigma^{2}}} ; x>0
$$

Its mean, variance, skewness are $\mu_{x}=\mathrm{e}^{\mu+\frac{\sigma^{2}}{2}}, \sigma_{x}^{2}=\left(\mathrm{e}^{\sigma^{2}}-1\right) \mathrm{e}^{2 \mu+\sigma^{2}}$, and $\gamma_{1}=\left(\mathrm{e}^{\sigma^{2}}-2\right) \sqrt{\mathrm{e}^{\sigma^{2}}-1}$ respectively. Mohan et al. [12] suggested a lognormal approximation of activity duration in PERT using two time estimates. His method handled the heavy tailed property of the activity time distribution which is deficient when normal activity time is assumed and also reduced the parameters from three (a-Optimistic, $m$-Most likely, and $b$ Pessimistic) to two (a-Optimis- 
tic, and $m$-Most likely) or ( $m$-Most likely, and $b$-Pessimistic). It was demonstrated with examples that their methods are better than the normal approximation when the underlying activity distribution is skewed to the right and better than the classical PERT method only when the activity distribution is heavily right skewed. Trietsch, et al. [49] suggested the use of lognormal distribution for modeling activity times but by the Parkinson effect distribution. They further considered that project activities exhibit stochastic dependence that can be modeled by linear association. Some theoretical and empirical justifications were presented as a justification for the use of the model. For more on lognormal activity time see Perry and Greig [30].

6) The Triangular Distribution

The triangular distribution has also been suggested as a priori distribution for activity times. Mac Crimmon and Ryavec [3] and Elmaghraby [5] earlier suggested that the triangular distribution could be considered as activity time distribution. The triangular distribution can be symmetric, positive or negative skewed. A random variable $X$ with triangular distribution has the probability density function,

$$
g(x)= \begin{cases}\frac{2}{b-a} \frac{x-a}{m-a} ; & a \leq x \leq m \\ \frac{2}{b-a} \frac{b-x}{b-m} ; & m \leq x \leq b\end{cases}
$$

where $m$ stands for the mode and the interval $[a, b]$ determines the range of the random variable $X$. The mean, variance and skewness are given as

$$
\mu_{x}=\frac{a+m+b}{3}, \sigma_{x}^{2}=\frac{a^{2}+b^{2}+m^{2}-a b-a m-b m}{18}
$$

and

$$
\gamma_{1}=\frac{\sqrt{2}(a+b-2 m)(2 a-b-m)(a-2 b+m)}{5\left(a^{2}+b^{2}+m^{2}-a b-a m-b m\right)}
$$

The $a, m$ and $b$ could be obtained intuitively as in the case of the classical PERT. Johnson [50] was interested in how a triangular distribution could be used in place of the beta distribution. His results showed that for a symmetric beta distribution, the triangular distribution can be used as a proxy with maximum deviation, $D=\operatorname{Max}\left|F\left(x_{i}\right)-G\left(x_{i}\right)\right|$, less than 0.03 , and greater than 0.02 when compared with extremely skewed beta distributions. Where $F\left(x_{i}\right)$ and $G\left(x_{i}\right)$ are beta and triangular distribution functions respectively. Williams [9] carried out an empirical assessment on the extent of bias of PERT beta (classical PERT and its modifications) models and PERT triangular model using simulation approach. His study revealed that the various modifications on classical PERT have not solved the problem of the intuitive adoption of beta distribution. See Hajdu and Bokor [8] and Okagbue, et al. [51] for more on triangular distribution.

7) The Uniform Distribution 
MacCrimmon and Rayvec [3] and (Elmaghraby [5] earlier suggested the use of uniform distribution as an activity time distribution based on two points estimates, the pessimistic and optimistic times and then the critical path method(CPM). A random variable $X$ defines activity duration on interval $[a, b]$ with probability density function given as $f(x)=\frac{1}{b-a} ; a<x<b$ with mean, variance, and skewness $\mu_{x}=\frac{a+b}{2}, \sigma_{x}^{2}=\frac{(b-a)^{2}}{12}$ and $\gamma_{1}=0$ respectively. Recently, Abdelkader and Al-Ohali [52] considered the problem of determining the project completion time when activity duration are uniform distributed using a recursive method, using two extreme points, $a$ and $b$ to be supplied by the expert. Their method followed after SANs technique. They opined that this method has an advantage over some activity task distributions with point estimates. For more work on uniform activity time distribution see Kleindorfer [53] and Hajdu and Bokor [8].

\section{8) The Erlang Distribution}

Bendell, et al. [54] developed the moments method based on Erlang activity time distribution. An Erlang distributed random variable $X$ has the probability density $f(x)=\frac{\lambda^{k}}{\Gamma(k)} \mathrm{e}^{-\lambda x} x^{k-1} ; x \geq 0 ; \lambda, k>0$.

Its mean, variance, and skewness are $\mu_{x}=\frac{k}{\lambda}, \sigma_{x}^{2}=\frac{k}{\lambda^{2}}$, and $\gamma_{1}=\frac{2}{\sqrt{k}}$ respectively. They obtained the first four central moments of the $\operatorname{Max}\left(X_{1}, X_{2}\right)$, where $X_{1}$ and $X_{2}$ are independent random variables, and further demonstrated the accuracy of their method in many practical scenario. Their method formed the basis upon which multi-modal activity time distributions could be used. Abdelkader [55] extended Bendell's work by obtaining the $K^{\text {th }}$ moments of the $\operatorname{Max}\left(X_{1}, X_{2}, \cdots, X_{n}\right)$ and the cumulative distribution function of the sum of $n$ independent random variables.

\section{9) The Gamma Distribution}

Lootsma [56] examined PERT and proposed a model for a project which every activity time follows a gamma distribution with density

$$
f(x)=\left\{\begin{array}{l}
\frac{\lambda^{\alpha}}{\Gamma(\alpha)}(x-a)^{\alpha-1} \exp \{-\lambda(x-a)\} ; x \geq a \\
0 ; x<a
\end{array}\right.
$$

where $\alpha$ is the shape parameter and $\lambda$ is the scale parameter of the gamma distribution. Its mean, variance and skewness are $\mu_{x}=\frac{\alpha}{\lambda}+a$ and $\sigma_{x}^{2}=\frac{\alpha}{\lambda^{2}}$ and $\gamma_{1}=\frac{2}{\sqrt{\alpha}}$ respectively. The estimates of the mean and variance were given as $\hat{\mu}_{x}=\frac{b-5 m}{6}$ and $\hat{\sigma}_{x}^{2}=\left(\hat{\mu}_{x}-m\right)\left(\hat{\mu}_{x}-a\right)$, based on intuitive time estimates (the optimistic (a), most likely $(m)$ and pessimistic $(b)$ times) from the practi- 
tioner. Abdelkader [57] also used the gamma distribution as an activity time distribution. His method followed after SANs. See Perry and Greig [30] for more on gamma activity time distribution.

10) The Compound Poisson distribution

Parks and Ramsing [58] considered the compound Poisson distribution for the activity times with the assumption that the minimum (pessimistic) time equals the most likely time. They were able to locate the joint probability of exactly $n$ arrivals from series of Poisson streams with different values and also capture the right skewed property in the data.

\section{1) The Beta Rectangular Distribution}

A mixture density, beta-rectangular distribution was introduced by Hahn [59] to approximate activity times in PERT. His intention was to introduce a distribution which permits varying amount of dispersion, instead of the constant variance provided by the classical PERT method. The beta rectangular mixture distribution was given as

$$
p(y, \alpha, \beta, \theta, a, b)=\frac{\theta \Gamma(\alpha+\beta)(y-a)^{\alpha-1}(b-y)^{\beta-1}}{\Gamma(\alpha) \Gamma(\beta)(b-a)^{\alpha+\beta-1}}+\frac{1-\theta}{b-a}
$$

where $\theta$ is the mixing parameter on interval $0 \leq \theta \leq 1$. The mean and variance of the mixture density are

$$
\mu_{y}=a+(b-a)\left(\frac{\theta \alpha}{k}+\frac{1-\theta}{2}\right)
$$

and

$$
\sigma_{y}^{2}=(a+b)^{2}\left(\frac{\theta \alpha(\alpha+1)}{k(k+1)}+\frac{1-\theta}{3}-\frac{(k+\theta(\alpha-\beta))^{2}}{4 k^{2}}\right)
$$

The mean and variance were approximated as

$$
\begin{gathered}
\hat{\mu}_{x}=\frac{\theta(a+4 m+b)+3(1-\theta)(a+b)}{6} \\
\hat{\sigma}_{x}^{2}=\frac{1}{36}\left[\theta\left((a+4 m+b)^{2}+(b-a)^{2}\right)+12(1-\theta)\left(a^{2}+a b+b^{2}\right)\right. \\
\left.-(\theta(a+4 m+b)+3(1-\theta)(a+b))^{2}\right]
\end{gathered}
$$

respectively. His method, in comparison with the classical PERT method, accommodated greater likelihood of more extreme tail- area events that seemed straight forward to implement with experts judgment. However, in addition to the three intuitive parameters $(a, m, b)$ of classical PERT, his method introduced the fourth parameter $\theta$ which should also be subjectively chosen by project managers. Yakhchali [60] proposed a method that could be used when project network consist of activities with different probability distributions. His approach consist of determining the exact cumulative distribution function of the earliest and latest starting and finishing and floats of activities based on the method of confidence interval. He demonstrated his method using both discrete 
and continuous probability distributions. Abou Rizkand Halpin [11] in an empirical study of construction duration data suggested the use of other flexible distributions like the Pearson and Johnson systems. The Pearson system and Johnson system cover almost the entire area of skewness and kurtosis plane.

\section{2) Tilted Beta Distribution}

Hahn and Martín [10] introduced the tilted beta distribution with probability density function

$$
\begin{aligned}
& p(x / v, \alpha, \beta, \theta) \\
& =\left\{\begin{array}{l}
(1-\theta)[2 v-2(2 v-1) x]+\theta\left[\frac{\Gamma(\alpha+\beta)}{\Gamma(\alpha) \Gamma(\beta)} x^{\alpha-1}(1-x)^{\beta-1}\right] ; 0 \leq x \leq 1 \\
0 ; \text { otherwise }
\end{array}\right.
\end{aligned}
$$

where $\theta \in[0,1]$ and $\theta \in[0,1]$. The mean and variance of the tilted beta distribution are

$$
(1-\theta) \frac{2-v}{3}+\theta \frac{\alpha}{\alpha+\beta}
$$

and

$$
\begin{aligned}
& \left((1-\theta) \frac{3-2 v}{6}-\theta \frac{\alpha(\alpha-1)}{(\alpha+\beta)(\alpha+\beta+1)}\right)-\left((1-\theta) \frac{2-v}{3}+\theta \frac{\alpha}{\alpha+\beta}\right)^{2} \\
& \left((1-\theta) \frac{3-2 v}{6}-\theta \frac{\alpha(\alpha-1)}{(\alpha+\beta)(\alpha+\beta+1)}\right)-\left((1-\theta) \frac{2-v}{3}+\theta \frac{\alpha}{\alpha+\beta}\right)^{2}
\end{aligned}
$$

respectively. The distribution is a mixture of the tilting distribution [61] and the beta distribution, with $\theta$ as the mixing parameter. The tilted beta distribution retains some known distributions as special cases. For instance, given the probability density of the tilted beta, if $\theta=1$ we have the beta distribution, if $\theta=0$ we obtain the tilted distribution, if $\theta=\frac{1}{2}$ either the beta distribution, uniform distribution, or beta rectangular distribution is obtained depending on the value of $v$. The parameters of the distribution where elicited as follows: Given the beta distribution with $k=\alpha+\beta=6 ; \alpha \neq \beta$ and noting that in this case the mean and mode are $\frac{\alpha}{k}$ and $\frac{\alpha-1}{k-2}$ respectively. Solving some simultaneous equations $\alpha$ and $\beta$ where recomputed as $4 m+1$ and $5+4 m$ for the standardized beta. To elicit $v$, it was assumed that there exists a linear increase or decrease in the probability density across time in accordance with the shape of the tilting distribution. Hence, the expert is requested to estimate the probability of the event of activity completion in day $j$ (say) denoted by $P\left(A_{j}\right)$ as well as the probability of the event of completion in day $j+1$, denoted by $P\left(A_{j+1}\right)$. Equating the rate of change denoted by $r=\frac{P\left(A_{j}\right)-P\left(A_{j+1}\right)}{1 /(b-a)} \quad$ ( $a=$ optimistic time, $b$ $=$ pessimistic time) to the slope of the tilted density function, $-2(2 v-1)$ and 
solving yields $v=\frac{2-r}{4}$. The mixing parameter $\theta$ was elicited as a judgmental estimate as in Hahn [59]. The tilted-beta distribution accommodates outlying events.

\section{3) Burr XII Distribution}

The Burr type 12 distribution [62] was found suitable for approximating activity times of water bore hole drilling project [63]. The Monte Carlo Simulation approach was adopted in conjunction with the classical PERT technique. This technique uses three judgmental estimates; pessimistic, most likely, and optimistic time estimates in the application of critical path algorithm to a long series of realization. Each activity time was obtained by assigning a sample value drawn from the Burr XII density. Results obtained from empirical studies showed that an error of $3 \%$ and $64 \%$ for mean and variance respectively would have occurred if the Beta distribution was used. The Burr XII density is positively skewed with much longer tail to accommodate outlying event. The distribution function Burr XII is closed, hence it allow for easy simulation. A random variable $X$ is said to follow Burr XII distribution with shape parameters, $c, k$, and a scale $\alpha$, if the probability density function is given as;

$$
f(x)=c k{\frac{x}{\alpha^{c}}}^{c-1}\left(1+\left(\frac{x}{\alpha}\right)^{c}\right)^{-(k+1)} ; x \geq 0, c>0, \alpha>0, k>0
$$

The cumulative distribution function is $F(x)=1-\left(1+\left(\frac{x}{\alpha}\right)^{c}\right)^{-k}$. The $r^{\text {th }}$ moment about the origin is given as

$$
E\left(X^{r}\right)=\frac{k \alpha^{r} \Gamma\left(k-\frac{r}{c}\right) \Gamma\left(\frac{r}{c}+1\right)}{\Gamma(k+1)} ; c k>r
$$

\section{Conclusions}

We have presented an up-to-date review of the activity time distributions used in PERT with highlights of various methods adopted for parameter estimation. From the review, three estimation approaches are outstanding, namely, Analytical Approximation, Monte Carlo Simulation and SANs, see Table 1 for details.

Monte Carlo Simulation has proved to be a versatile technique with regards to the choice of distributional forms. Apart from the exact technique, the simulation technique has the capacity to produce more efficient results PMBOK [15]. However, the application of Monte Carlo Simulation approach suffers a set back because most of the activity time distributions are not listed in the available simulation packages. Another possible reason for the scanty use of the simulation technique is because the distributional form of some of the activity time distributions is not closed. The extent of simulation technique usage can be verified in column 2 of Table 1. 
Table 1. Summary of activity time distributions used in project network analysis.

\begin{tabular}{|c|c|}
\hline Probability Distribution & Method of Estimation \\
\hline Beta & $\begin{array}{l}\text { Analytical approximation. } \\
\text { Monte Carlo Simulation. }\end{array}$ \\
\hline Normal & $\begin{array}{c}\text { Analytical approximation. } \\
\text { Analytical bounding. } \\
\text { Exact analysis. }\end{array}$ \\
\hline Exponential & SANs \\
\hline Weibull & $\begin{array}{c}\text { Analytical approximation. } \\
\text { SANs }\end{array}$ \\
\hline Lognormal & Analytical approximation \\
\hline Triangular & $\begin{array}{l}\text { Analytical approximation. } \\
\text { Monte Carlo Simulation. }\end{array}$ \\
\hline Uniform & $\begin{array}{l}\text { Analytical approximation. } \\
\text { SANs. } \\
\text { Monte Carlo Simulation. }\end{array}$ \\
\hline Erlang & SANs. \\
\hline Gamma & $\begin{array}{c}\text { Analytical approximation. } \\
\text { SANs. }\end{array}$ \\
\hline Compound Poisson & Analytical approximation \\
\hline Beta-Rectangular & Analytical approximation. \\
\hline Tilted-Beta & Analytical approximation. \\
\hline Burr XII & Monte Carlo Simulation \\
\hline
\end{tabular}

A basic advantage of the Simulation approach is that it allows the use of any activity time distribution. In short, different distributions can be used on different activities of the same project. It was observed that the choice of most of the activity time distributions was based on flexibility and convenience, with no clear empirical evidences, as earlier noted by Trietsch et al. [49]. This review also points to the fact that the beta distribution is not the sole activity time distribution as presented in most basic texts and lecture notes on project managements.

The importance of appropriate choice of activity time distribution cannot be overemphasized, irrespective of the method adopted to estimate the parameters of project network. Hence, we suggest that practitioners, apart from using theoretical information, should endeavor to make their choices of activity duration distributions based on particular empirical evidences and not just on simplicity. Developers of project management software should also incorporate many probability distributions as much as possible to enable users' flexibility of choice. The information provided in this research can be used to extend the study by Hajdu and Bokor [8].

\section{References}

[1] Pierce, D. (2006) Project Scheduling and Management for Construction. 3rd Edition, Reed Construction Data, Inc., Kingston, MA. 
[2] Malcom, D., Roseboom, J. and Clark, C. (1959) Application of a Technique for Research and Development Program Evaluation. Operations Research, 7, 646-669. https://doi.org/10.1287/opre.7.5.646

[3] MacCrimmon, K. and Ryavec, C.A. (1964) An Analytical Study of the PERT Assumptions. Operations Research, 12, 16-37. https://doi.org/10.1287/opre.12.1.16

[4] Lootsma, F. (1989) Stochastic and Fuzzy PERT. European Journal of Operational Research, 43, 174-183. https://doi.org/10.1016/0377-2217(89)90211-7

[5] Elmaghraby, S.E. (1977) Activity Networks: Project Planning and Control by Networks. John Wiley \& Sons, New York.

[6] Clark, C.E. (1962) The PERT Model for the Distribution of an Activity Time. Operations Research, 10, 405-406. https://doi.org/10.1287/opre.10.3.405

[7] Kamburowski, J. (1997) New Validations of PERT Times. International Journal of Medical Sciences, 25, 323-328. https://doi.org/10.1016/S0305-0483(97)00002-9

[8] Hajdu, M. and Orsolyo, B. (2014) The Effects of Different Activity Distributions on Project Duration in PERT Networks. Procedia Social and Behavioural Sciences, 119, 766-775. https://doi.org/10.1016/j.sbspro.2014.03.086

[9] Williams, T.M. (1992) PERT Completion Times Revisited. INFORMS Transactions in Education, 6, 21-34. https://doi.org/10.1287/ited.6.1.21

[10] Hahn, E.D. and Martín, M.M.L. (2015) Robust Project Management with the Tilted Beta Distribution. SORT, 39, 253-272.

[11] Abou Rizk, S. and Halpin, D. (1992) Statistical Properties of Construction Duration Data. Journal of Construction Engineering Management, 118, 525-544. https://doi.org/10.1061/(ASCE)0733-9364(1992)118:3(525)

[12] Mohan, S., Gopalakrishnan, M., Balasubramanian, H. and Chandrashekar, A. (2007) A Lognormal Approximation of Activity Duration in PERT Using Two Time Estimates. Journal of the Operational Research Society, 58, 827-831. https://doi.org/10.1061/(ASCE)0733-9364(1992)118:3(525)

[13] Shankar, N.R., Babu, S.S., Thorani, Y.L.P. and Raghuram, D. (2011) Right Skewed Distribution of Activity Times in PERT. International Journal of Engineering Science and Technology, 3, 2932-2938.

[14] Van Slyke, R. (1963) Monte Carlo Methods and the PERT Problems. Operations Research, 11, 839-860. https://doi.org/10.1287/opre.11.5.839

[15] PMBOK (2008) A Guide to the Project Management Body of Knowledge. 4th Edition, Project Management Institute Inc., Newtown Square, Pennsylvania.

[16] Farnum, N. and Stanton, L. (1987) Some Results Concerning the Estimation of Beta Distribution Parameters in PERT. Journal of the Operational Research Society, 38, 287-290. https://doi.org/10.1057/jors.1987.45

[17] Grubbs, F.E. (1962) Attempts to Validate Certain PERT Statistics or Picking on PERT. Operations Research, 10, 912-915. https://doi.org/10.1287/opre.10.6.912

[18] Premachandra, M. (2001) An Approximation of The Activity Duration Distribution in PERT. Computing and Operations Research, 28, 443-452. https://doi.org/10.1016/S0305-0548(99)00129-X

[19] Herrerias-Velasco, J.M., Herrerias-Pleguezuelo, R. and Dorp, J.R.V. (2011) Revisiting the PERT Mean and Variance. European Journal of Operational Research, 210, 448-451. https://doi.org/10.1016/j.ejor.2010.08.014

[20] Golenko-Ginzburg, D. (1988) On the Distribution of Activity Times in PERT. Journal of the Operational Research Society, 39, 767-771. 
https://doi.org/10.1057/jors.1988.132

[21] Nowpada, R.S. and Veeramachaneni, S. (2009) An Approximation for the Activity Duration Distribution, Supporting Original PERT. Applied Mathematical Sciences, 57, 2823-2834.

[22] Trout, M.D. (1989) On the Generality of the PERT Average Time Formula. Decision Sciences, 20, 491-412. https://doi.org/10.1111/j.1540-5915.1989.tb01888.x

[23] Arsham, H. (1993) Managing Project Duration Uncertainties. Omega, 21, 111-122. https://doi.org/10.1016/0305-0483(93)90043-K

[24] Donaldson, W.A. (1985) Estimation of Mean and Variance of a PERT Network. Operations Research, 33, 862-881.

[25] Gallagher, C. (1987) A Note on PERT Assumptions. Management Science, 33, 1360. https://doi.org/10.1287/mnsc.33.10.1360

[26] Healy, T.H. (1961) Activity Subdivision and PERT Probability Statements. Operations Research, 9, 341-348. https://doi.org/10.1287/opre.9.3.341

[27] Littlefield, T.K. and Randolph, P.H. (1987) An Answer to Sasieni's Question on PERT Times. Managements Science, 33, 1357-1359. https://doi.org/10.1287/mnsc.33.10.1357

[28] Moder, J.J. and Rodgers, E.G. (1968) Judgment Estimates of the Moments of PERT Type Distributions. Management Science, 15, 76-83. https://doi.org/10.1287/mnsc.15.2.B76

[29] Sasieni, M.V. (1986) A Note on PERT Times. Management Sciences, 32, 1652-1653. https://doi.org/10.1287/mnsc.32.12.1652

[30] Perry, C. and Greig, I.D. (1975) Estimating the Mean and Variance of Subjective Distributions in PERT and Decision Analysis. Management Sciences, 21, 1477-1480. https://doi.org/10.1287/mnsc.21.12.1477

[31] Keefer, D.L. and Verdini, W.A. (1993) Better Estimation of PERT Activity Time. Management Sciences, 39, 1086-1091. https://doi.org/10.1287/mnsc.39.9.1086

[32] Kamburowski, J. (1985) Normally Distributed Activity Durations in PERT Networks. Journal of the Operational Research Society, 36, 1051-1057. https://doi.org/10.1057/jors.1985.184

[33] Dodin, B. (1985) Bounding the Project Completion Time Distribution in PERT Networks. Operations Research, 33, 862-881. https://doi.org/10.1287/opre.33.4.862

[34] Sculli, D. (1983) The Completion Time of PERT. Journal of Operational Research Society, 34, 155-158. https://doi.org/10.1057/jors.1983.27

[35] Drezner, Z. and Anklesaria, K.P. (1986) A Multivariate Approach to Estimating the Completion Time for PERT Networks. Journal of Operational Research Society, 37, 811-815. https://doi.org/10.1057/jors.1986.140

[36] Cottrell, P.E. and Wayne, D. (1999) Simplified Program Evaluation and Review Technique (PERT). Journal of Construction Engineering and Management, 125, 16-22. https://doi.org/10.1061/(ASCE)0733-9364(1999)125:1(16)

[37] Kotiah, T.C.T. and Wallace, N.D. (1979) Another Look at PERT Assumptions. Management Sciences, 20, 44-49. https://doi.org/10.1287/mnsc.20.1.44

[38] Maggot, J. and Skudlarski, K. (1993) Estimating the Mean Completion Time of PERT Network with Exponentially Distributed Duration of Activities. European Journal of Operational Research, 71, 70-79. https://doi.org/10.1016/0377-2217(93)90261-K

[39] Abdelkader, Y.H. and Mouhamed, M.S. (1998) Exponentially Distributed Activity 
Duration for Stochastic Acyclic Networks. Journal of Indian Society of Statistics and Operations Research, 19, 37-49.

[40] Abdelkader, Y.H. (2010) Adjustment of the Moments of the Project Completion Times when Activity Times are Exponentially Distributed. Annals of Operations Research, 181, 503-514. https://doi.org/10.1007/s10479-010-0781-3

[41] Abdelkader, Y. (2006) Stochastic Activity Networks with Truncated Exponential Activity Times. Journal of Applied Mathematics and Computing, 20, 119-132. https://doi.org/10.1007/BF02831927

[42] Cinicioglu, E.N. and Shenoy, P.P. (2009) Using Mixtures of Truncated Exponentials for Solving Stochastic PERT Networks. Proceedings of the Eighth Workshop on Uncertainty Processing (WUPES 09), Liblice, 19-23 September 2009, 269-283.

[43] Azaron, A. and Modarres, M. (2007) Project Completion Time in Dynamic PERT Networks with Generating Projects. Scientia Iranica, 14, 56-63.

[44] Kamburowski, J. (1985b) An Upper Bound on the Expected Completion Time of PERT Networks. European Journal of Operational Research, 21, 206-212. https://doi.org/10.1016/0377-2217(85)90032-3

[45] Kulkarni, V.G. and Adlakha, V.G. (1986) Markov and Markov Regenerative PERT Networks. Operations Research, 34, 769-781. https://doi.org/10.1287/opre.34.5.769

[46] Kwon, H.D., Lippman, A.S., McCCardle, K.F. and Tang, C.S. (2010) Project Management Contracts with Delayed Payment. Mamufacturing and Service Operations Research, 12, 692-707. https://doi.org/10.1287/msom.1100.0301

[47] Abdelkader, Y.H. (2004) Evaluating Project Completion Times When Activity Times Are Webull Distributed. European Journal of Operational Research, 157, 704-715. https://doi.org/10.1016/S0377-2217(03)00269-8

[48] McCombs, E.L., Elam, M.E. and Pratt, D.B. (2009) Estimating Task Duration in PERT Using the Weibull Probability Distribution. Journal of Modern Applied Statistical Methods, 8, 282-288. https://doi.org/10.22237/jmasm/1241137500

[49] Trietsch, D., Mazmanyan, L., Gevorgyan, L. and Baker, K.R. (2012) Modeling Activity Times by the Parkinson Distribution with a Lognormal Core: Theory and Validation. European Journal of Operational Research, 216, 386-396. https://doi.org/10.1016/j.ejor.2011.07.054

[50] Johnson, D. (1997) The Triangular Distribution as a Proxy for Beta Distribution in Risk Analysis. The Statistician, 46, 387-398. https://doi.org/10.1111/1467-9884.00091

[51] Okagbue, H.I., Edeki, S.O., Opanugu, A.A., Oguntunde, P.E. and Adeosun, M.E. (2014) Using the Average of the Extreme Values of a Triangular Distribution for a Transformation, and Its Approximant via the Continuous Uniform Distribution. British Journal of Mathematics and Computer Science, 4, 3497-3507. https://doi.org/10.9734/BJMCS/2014/12299

[52] Abdelkader, Y.H. and Al-Ohali, M. (2013) Estimating the Completion Time of Stochastic Activity Networks with Uniform Distributed Activity Times. Archives Des Sciences, 66, 115-134.

[53] Kleindorfer, G.B. (1971) Bounding Distributions for a Stochastic Acyclic Network. Operations Research, 19, 1586-1601. https://doi.org/10.1287/opre.19.7.1586

[54] Bendell, A., Solomon, D. and Carter, J.M. (1995) Evaluating Project Completion Times When Activity Times Are Erlang Distributed. Journal of Operational Research Society, 46, 867-882. https://doi.org/10.1057/jors.1995.118

[55] Abdelkader, Y.H. (2003) Erlang Distributed Activity Times in Stochastic Activity 
Networks. Kybernetica, 39, 347-358.

[56] Lootsma, F.A. (1966) Network Planning with Stochastic Activity Durations: An Evaluation of PERT. Statistica Neerlandica, 20, 43-69.

https://doi.org/10.1111/j.1467-9574.1966.tb00492.x

[57] Abdelkader, Y.H. (2004(b)) Computing the Moments of Order Statistics from Nonidentically Distributed Gamma Variables with Applications. International Journal of Mathematics, Game Theory and Algebra, 14, 1-8.

[58] Parks, W. and Ramsing, K. (1969) The Use of Compound Poisson in PERT. Management Science, 15, 397-402. https://doi.org/10.1287/mnsc.15.8.B397

[59] Hahn, E. (2008) Mixture of Densities for Project Management Activity Times: A Robust Approach to PERT. European Journal of Operational Research, 18; 450-459. https://doi.org/10.1016/j.ejor.2007.04.032

[60] Yachchali, S.H. (2011) A Simple Approach to Project Scheduling in Networks with Stochastic Durations. Proceedings of the World Congress on Engineering, 1, London, 6-8 July 2011.

[61] Topp, C.W. and Leone, F.C. (1955) A Family of J-Shaped Frequency Functions. Journal of the American Statistical Association, 50, 209-219. https://doi.org/10.1080/01621459.1955.10501259

[62] Burr, I.W. (1942) Cumulative Frequency Functions. Annals of Mathematical Statistics, 13, 215-232. https://doi.org/10.1214/aoms/1177731607

[63] Udoumoh, E.F., Ebong, D.W. and Iwok, I.A. (2017) Simulation of Project Completion Time with Burr XII Activity Distribution. ARJOM, 6, 1-14.

https://doi.org/10.9734/ARJOM/2017/35707 